\begin{abstract}
Mrs Tan took her three-day-old child, Michael, to your clinic for a review of his neonatal jaundice. She provided a letter of discharge from the hospital where Michael was delivered. You reviewed the child's health booklet and noticed that his total serum bilirubin level, from the afternoon of the day before their visit, was $119 \mu \mathrm{mol} / \mathrm{L}(7 \mathrm{mg} / \mathrm{dL})$. You asked how Michael had adjusted and how everyone at home had adapted to their new roles since the birth. Mrs Tan wanted advice on what else she could do to improve Michael's jaundice and whether exposure to the sun would help.
\end{abstract}

\section{WHAT IS NEONATAL JAUNDICE?}

Jaundice refers to a condition caused by the accumulation of bilirubin in the skin, mucous membranes and sclerae. Patients present with yellow colouration of the skin and whites of the eyes. Neonatal jaundice, which refers to jaundice during the early days of life, has many aetiologies, including maternal and fetal blood group incompatibility (most commonly Rhesus or $\mathrm{ABO}$ incompatibility), certain causes of haemolysis (e.g. resolving cephalohaematoma), infections, liver disease, bruising and metabolic disorders. ${ }^{(1)}$ Glucose-6-phosphate dehydrogenase (G6PD) deficiency is one common cause of severe neonatal jaundice in Singapore. ${ }^{(2)}$ Bilirubin is mainly produced by the breakdown of red blood cells. Most of the unconjugated (or 'indirect') bilirubin formed is bound to albumin as it circulates, although some bilirubin is 'free' and hence able to enter the brain. Unconjugated bilirubin is metabolised in the liver to produce conjugated (or 'direct') bilirubin, which passes into the gut and is largely excreted in stool. ${ }^{(1)}$

\section{HOW RELEVANT IS THIS TO MY PRACTICE?}

Raised bilirubin levels in the body, or hyperbilirubinaemia, is common in the first week of life, affecting $50 \%-70 \%$ of term babies and $80 \%$ of preterm babies. ${ }^{(3-5)}$ Up to $30 \%-40 \%$ of predominantly breastfed babies may still be jaundiced at the age of 3-4 weeks. ${ }^{(6)}$ Breast milk jaundice is a common type of unconjugated hyperbilirubinaemia ${ }^{(7,8)}$ that may occur in the second or third week of life. It is thought to be due to metabolites in breast milk that reduce the hepatic uptake of unconjugated bilirubin and increase enterohepatic reuptake. Breast milk jaundice should be differentiated from breastfeeding jaundice, which occurs earlier in the child's life and is due to poor milk intake, resulting in an exaggerated physiological response. ${ }^{(8)}$ The incidence of neonatal jaundice in Asian populations is increasing, possibly due to a higher incidence of gene mutations, which result in reduced function of the uridine 5'-diphosphoglucuronosyltransferase enzyme that is responsible for bilirubin conjugation. ${ }^{(9,10)}$

Most cases of neonatal jaundice are physiological, with no signs of an underlying disease. The hyperbilirubinaemia is predominantly unconjugated ${ }^{(1)}$ and generally harmless. However, when the levels of unconjugated bilirubin are high, it can cross the blood-brain barrier and cause both short- and long-term neurological dysfunction (i.e. kernicterus or bilirubin encephalopathy). Jaundice in newborns can also have pathological causes, which although rare, must be detected. Thus, the role of the primary care practitioner is to: (a) evaluate the jaundiced neonate to rule out pathological causes; and (b) monitor physiological jaundice and refer the child to tertiary care if his/her bilirubin approaches dangerous levels.

\section{WHAT CAN I DO IN MY PRACTICE? Primary prevention}

The primary care practitioner should promote successful breastfeeding, as poor caloric intake and/or dehydration may contribute to the development of neonatal jaundice. ${ }^{(11)}$ To assess feeding, clinical history should include an evaluation of latching technique and whether the child feeds at a frequency of 8-12 times a day for the first few days of life. Adequate formula feeding is approximately $150 \mathrm{kcal} / \mathrm{kg} /$ day, i.e. about $1-2 \mathrm{oz}$ every $2-3$ hours, for an infant with average birth weight. Routine supplementation with water is neither necessary nor beneficial to prevent hyperbilirubinaemia or treat neonatal jaundice.

\section{Clinical assessment}

Clinical recognition of neonatal jaundice by visual inspection can be difficult, particularly in babies with darker skin tones. Clinical inspection for jaundice should be performed within the first 48 hours of life by a healthcare professional, usually a neonatologist or family doctor in Singapore. It should be 


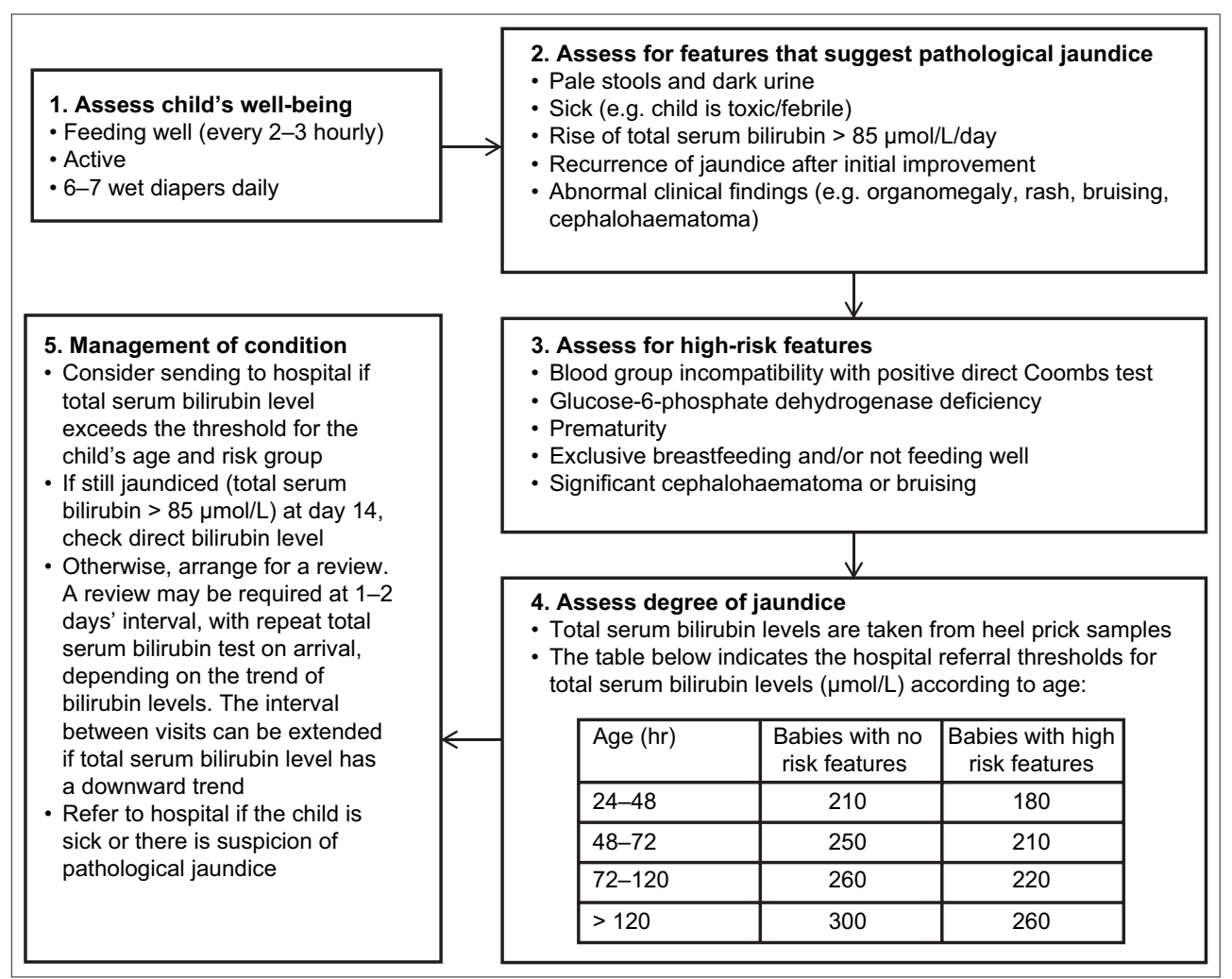

Fig. 1 Flowchart shows an overview of the management of neonatal jaundice.

performed under bright and, if possible, natural light. The visual examination should include the sclerae, gums and blanched skin of the limbs, torso and forehead. Fig. 1 shows the steps that can be taken to identify and manage neonatal jaundice.

\section{Assessment of the child's well-being}

An important aspect of the initial assessment is to ascertain if the child is well. This would include history that the child is active and feeding well (i.e. every 2-3 hours) and well-hydrated (i.e. at least 6-7 wet diapers daily). Any sick child should be referred to hospital immediately.

\section{Assessment for features that suggest pathological jaundice}

Apart from physiological neonatal jaundice and those related to breastfeeding, all other causes of neonatal jaundice are pathological. Features of pathological jaundice include: ${ }^{(8)}$

- Jaundice within 24 hours of life;

- $\quad$ Rise in total serum bilirubin level of more than $85 \mu \mathrm{mol} / \mathrm{L}(5 \mathrm{mg} / \mathrm{dL})$ per day;

- Total serum bilirubin level exceeding $290 \mu \mathrm{mol} / \mathrm{L}$ $(17 \mathrm{mg} / \mathrm{dL})$ in a term baby;

- $\quad$ Conjugated hyperbilirubinaemia, as evidenced by a raised direct bilirubin level or the presence of dark urine and pale stools - the parent or carer should be asked about stool colour; if conjugated jaundice is suspected, the direct bilirubin level should be measured and hospital referral strongly considered;

- Abnormal clinical findings - the abdomen should be palpated to check for organomegaly and the skin carefully inspected for rash, bruising and cephalohaematoma, watching out for features that suggest intrauterine infections such as microcephaly, cataracts, etc;

- Other features that would point to pathological jaundice, including findings of deep jaundice and recurrence of jaundice after initial improvement.

\section{Assessment for high-risk features}

Children with high-risk features for jaundice are at risk of bilirubin encephalopathy, even when total serum bilirubin levels are relatively lower; ${ }^{(1)}$ therefore, the threshold for referral to hospital is lower for children. High-risk features ${ }^{(11)}$ include:

- Blood group incompatibility (as seen in a positive direct Coombs test);

- G6PD deficiency;

- $\quad$ Premature birth at 35-36 weeks;

- $\quad$ Exclusive breastfeeding and/or not feeding well;

- $\quad$ Significant cephalohaematoma or bruising.

Apart from history and physical examination, a good way to gather this data would be to peruse the child's health booklet. This booklet, which is given to most children in Singapore, documents information pertinent to the assessment of neonatal jaundice such as the child's cord thyroid-stimulating hormone results, birth history (e.g. duration of pregnancy, birth weight, APGAR scores, etc), G6PD results and vaccination history.

\section{Assessment of the degree of neonatal jaundice}

All babies with clinical jaundice should have their total serum bilirubin level measured to obtain actual measurements rather 
than rely on visual inspection alone. ${ }^{(11)}$ Laboratory test options in primary care include measurements of total and direct bilirubin levels. When laboratory testing is not available, an alternative option is a referral to the nearest polyclinic. Total serum bilirubin levels from heel prick samples should be taken at intervals; the frequency and intervals for measurements depend on the clinical trend and rate of increase or decrease. A downward trend is reassuring. All bilirubin levels should be interpreted according to the infant's age in hours. ${ }^{(11)}$ Primary care practitioners in Singapore can refer to guidelines on the total serum bilirubin level at which the neonate should be referred to tertiary care for phototherapy. ${ }^{(12)}$ Total serum bilirubin thresholds for referral to hospital for further management are summarised in Fig. 1 (see Table).

\section{Approach to prolonged neonatal jaundice}

Jaundice persisting beyond 14 days of age (i.e. prolonged jaundice) can be a sign of serious underlying liver disease. ${ }^{(13,14)}$

\section{Measurement of direct bilirubin}

At the primary care level, the primary aim is to check for cholestatic jaundice, all cases of which should be referred to a hospital. Clinically, most patients with cholestatic liver disease have jaundice, dark urine and pale stools, but otherwise appear healthy. The measurement of conjugated ('direct') bilirubin is the most important initial investigation. Total serum bilirubin and direct bilirubin levels should be tested in every term baby who has jaundice that lasts beyond 14 days. ${ }^{(14)}$ Conjugated hyperbilirubinaemia may be defined based on the absolute value of the child's direct bilirubin level (more than $35 \mu \mathrm{mol} / \mathrm{L}$ ) or its relative value (more than $15 \%$ of total serum bilirubin). ${ }^{14,15}$ Cholestatic jaundice has many causes, but extrahepatic biliary atresia and neonatal hepatitis account for $70 \%-80 \%$ of cases of conjugated jaundice; ${ }^{(16)}$ urinary tract infection may be another consideration. ${ }^{(11)}$ Detailed evaluation for cholestatic jaundice is beyond the scope of this article and not discussed here.

\section{Inspection of stool colour}

As there is no internationally agreed level of serum bilirubin for biliary atresia, it cannot be excluded with complete confidence. The most promising strategies are early measurements of serum conjugated bilirubin and assessment of stool colour. ${ }^{(17)}$ Hence, reviews of babies who have neonatal jaundice beyond 14 days of life must include assessments of stool colour. ${ }^{(1,18)}$ The use of stool colour charts may help to increase parental and physician awareness and aid in the earlier referral of babies with biliary atresia. ${ }^{(19-21)}$ Ideally, physicians should request to personally inspect the stool. Confirming stool colour through photography or actual inspection is important, as parents may not be able to recognise pale stools. ${ }^{(22)}$ When following up with babies with prolonged neonatal jaundice, a practical tip for stool inspection is to ask the parent to collect the stool specimen the day before the appointment. If the child continues to have bowel movement, the parent should discard the old sample in favour of the new specimen. This is done to avoid instances where the child does not move his/her bowels on the day of the follow-up. Healthcare professionals should be familiar with the colour of normal and abnormal stools. A useful resource is the Yellow Alert stool chart from the Children's Liver Disease Foundation. ${ }^{(23)}$

\section{Opportunistic screening at one month}

At one month of age, many babies see their family doctors for their second dose of hepatitis B vaccine. ${ }^{(24)}$ This presents another opportunity for doctors and nurses to perform a visual assessment under bright and, preferably, natural light to identify prolonged neonatal jaundice or late-onset neonatal jaundice.

\section{Patient-centred care}

The primary care practitioner plays an important role in educating parents and allaying their concerns. Parents may not be aware that early-onset jaundice (within the first 24 hours of life) is abnormal and may follow the lay advice of exposing jaundiced neonates to direct sunlight. ${ }^{(25-27)}$ They should be told that neonatal jaundice is common, usually transient and harmless. Parents should also be taught to look out for pathological patterns, such as jaundice appearing within the first 24 hours of the child's life and the associated signs of dark urine and pale stools. Education should include the importance of maintaining good hydration and that breastfeeding can usually continue.

\section{Referral to a specialist}

Any baby with poor nutrition or nonreassuring history should be referred early for assessment. Likewise, all conjugated hyperbilirubinaemia should be referred. Referral can be made to general paediatric clinics, gastroenterology clinics or the Children's Emergency. An example of a case that requires urgent review is that of a one-month-old child with hyperbilirubinaemia, in whom biliary atresia is suspected. A critical factor in the success of Kasai portoenterostomy is the age at which surgery is performed. ${ }^{(28)}$

You explained the concept of physiological neonatal jaundice to Mrs Tan and explicated the danger signs to look out for. You encouraged her to continue total breastfeeding (at least for the entire duration of her maternity leave) and instructed her to return to your clinic if she noticed her child passing any pale stools in the next few weeks. Over the next two to three weeks, you reviewed Michael in your clinic a few times. On each visit, you assessed him clinically and ordered total serum bilirubin tests. Michael's total serum bilirubin level showed a gradual increase toward the second week of life, stabilised at around $170 \mu \mathrm{mol} / \mathrm{L}$ $(10 \mathrm{mg} / \mathrm{dL})$ at week 3 , then showed a definite downward trend toward one month of life. A direct bilirubin test performed at 14 days of life found that the level was not elevated. By the age of one month, Michael was no longer jaundiced and had a total serum bilirubin level of $50 \mu \mathrm{mol} / \mathrm{L}(3 \mathrm{mg} / \mathrm{dL})$. 


\section{TAKE HOME MESSAGES}

1. Parents should be given advice on primary prevention measures such as proper breastfeeding and ensuring adequate hydration.

2. The general well-being of a jaundiced neonate should be assessed and any baby who is sick or feeding poorly should be promptly referred to hospital.

3. Features of pathological jaundice include early onset within 24 hours of life, rapid rise of total serum bilirubin level, pale stools or a raised direct bilirubin level.

4. Total serum bilirubin should be measured at intervals and interpreted according to the child's age in hours.

5. High-risk features such as prematurity, blood group incompatibility and G6PD deficiency would lower the threshold for referral to hospital.

6. Direct bilirubin measurements should be taken in babies with prolonged jaundice (persisting beyond 14 days of age). Levels exceeding $35 \mu \mathrm{mol} / \mathrm{L}$ or $15 \%$ of total serum bilirubin are considered abnormal.

7. Stool inspection is an important aspect of assessment. Parents should be educated on recognising stool colour and given a stool colour chart.

8. Visual assessment (under bright, natural lighting) and stool colour assessment should be performed again at one month of age, for babies who are still jaundiced.

9. Biliary atresia and neonatal hepatitis are causes of a significant majority of cases of cholestatic jaundice.

10. All babies suspected to have cholestatic jaundice should be seen early at the tertiary setting, as time is a critical factor in the success of Kasai portoenterostomy.

\section{REFERENCES}

1. National Institute for Health and Care Excellence. Neonatal jaundice: NICE guidelines [CG98] [online]. Available at: https://www.nice.org. uk/guidance/cg98/chapter/guidance\#management-and-treatment-ofhyperbilirubinaemia. Accessed October 17, 2015.

2. Shah VA, Yeo CL. Identifying risk of neonatal hyperbilirubinaemia and early discharge for glucose-6-phosphate dehydrogenase deficient newborns in Singapore. Ann Acad Med Singapore 2007; 36:1003-9.

3. Sarici SU, Serdar MA, Korkmaz A, et al. Incidence, course, and prediction of hyperbilirubinemia in near-term and term newborns. Pediatrics 2004; 113:775-80.

4. Cohen SM. Jaundice in the full-term newborn. Pediatr Nurs 2006; 32:202-8.

5. Newman TB, Xiong B, Gonzales VM, Escobar GJ. Prediction and prevention of extreme neonatal hyperbilirubinemia in a mature health maintenance organization. Arch Pediatr Adolesc Med 2000; 154:1140-7.

6. Maisels MJ, Clune S, Coleman K, et al. The natural history of jaundice in predominantly breastfed infants. Pediatrics 2014; 134:e340-5.

7. Auerbach KG, Gartner LM. Breastfeeding and human milk: their association with jaundice in the neonate. Clin Perinatol 1987; 14:89-107.

8. Porter ML, Dennis BL. Hyperbilirubinemia in the term newborn. Am Fam Physician 2002; 65:599-606.

9. Setia S, Villaveces A, Dhillon P, Mueller BA. Neonatal jaundice in Asian, white, and mixed-race infants. Arch Pediatr Adolesc Med 2002; 156:276-9.

10. Zhou YY, Lee LY, Ng SY, et al. UGT1A1 haplotype mutation among Asians in Singapore. Neonatology 2009; 96:150-5.

11. American Academy of Pediatrics Subcommittee on Hyperbilirubinemia. Management of hyperbilirubinemia in the newborn infant 35 or more weeks of gestation. Pediatrics 2004; 114:297-316.
ABSTRACT Neonatal jaundice is a common condition seen in the primary care setting. Most afflicted babies have physiological jaundice and their prognosis is good. However, others have pathological jaundice, which must be detected early. High levels of serum bilirubin can also result in bilirubin encephalopathy. This article describes consultation tasks in the primary care setting with the aim of providing a guide for the safe management of neonatal jaundice. They include clinical assessment of the baby's well-being; looking out for features that suggest pathological jaundice; assessment for the presence of high-risk features; utilising appropriate laboratory tests for monitoring; assessing the degree of jaundice to decide if the child can be safely followed up in primary care; and providing advice on primary prevention measures and allaying parental concerns. The importance of stool colour examination and its role in early detection of cholestatic jaundice is emphasised.

Keywords: neonatal jaundice, primary care

12. KK Women \& Children's Hospital. Department of Emergency Medicine Clinical Guidelines. Singapore: KK Women \& Children's Hospital, 2015.

13. Hannam S, McDonnell M, Rennie JM. Investigation of prolonged neonatal jaundice. Acta Paediatr 2000; 89:694-7.

14. Gilmour SM. Prolonged neonatal jaundice: When to worry and what to do. Paediatr Child Health 2004; 9:700-4

15. Karpen SJ. Update on the etiologies and management of neonatal cholestasis. Clin Perinatol 2002; 29:159-80.

16. El-Youssef M, Whitington P. Diagnostic approach to the child with hepatobiliary disease. Semin Liver Dis 1998; 18:195-202.

17. Sokol RJ, Shepherd RW, Superina R, et al. Screening and outcomes in biliary atresia: summary of a National Institutes of Health workshop. Hepatology 2007; 46:566-81.

18. Hartley JL, Davenport M, Kelly DA. Biliary atresia. Lancet 2009; 374:1704-13.

19. Chen SM, Chang MH, Du JC, et al; Taiwan Infant Stool Color Card Study Group. Screening for biliary atresia by infant stool color card in Taiwan. Pediatrics 2006; 117:1147-54.

20. Gu YH, Yokoyama K, Mizuta K, et al. Stool color card screening for early detection of biliary atresia and long-term native liver survival: a 19-year cohort study in Japan. J Pediatr 2015; 166:897-902.e1.

21. Tseng JJ, Lai MS, Lin MC, Fu YC. Stool color card screening for biliary atresia. Pediatrics 2011; 128:e1209-15.

22. Ho SS, Haller W, Catto-Smith AG. Yellow is pale: the complications and challenges of late diagnosis of extrahepatic biliary atresia. J Paediatr Child Health 2013; 49:152-4; quiz 154-5.

23. Children's Liver Disease Foundation. Stool Chart [online]. Available at: http://www.yellowalert.org/file_download.aspx?id=7358. Accessed October 21, 2015

24. Ministry of Health, Singapore. National Childhood Immunisation Schedule, Singapore [online]. Available from: https://www.moh.gov.sg/content/dam/ moh_web/Diseases\%20and\%20Conditions/Influenza/NCIS\%201\%20 June\%202013.pdf. Accessed October 21, 2015.

25. Aladag N, Filiz TM, Topsever P, Gorpelioglu S. Parents' knowledge and behaviour concerning sunning their babies; a cross-sectional, descriptive study. BMC Pediatr 2006; 6:27.

26. Poon WB, Ho WL, Yeo CL. Survey on parenting practices among Chinese in Singapore. Singapore Med J 2007; 48:1006-11.

27. Boo NY, Gan CY, Gian YW, et al. Malaysian mothers' knowledge \& practices on care of neonatal jaundice. Med J Malaysia 2011; 66:239-43.

28. Wildhaber BE. Biliary atresia: 50 years after the first kasai. ISRN Surg 2012; 2012:132089 


\section{SINGAPORE MEDICAL COUNCIL CATEGORY 3B CME PROGRAMME} (Code SMJ 201511A)

1. Neonatal jaundice refers to a condition, caused by the accumulation of carotene or bilirubin in the skin, mucous membranes and sclerae, in which patients present with yellow colouration of the skin and whites of the eyes.

2. Neonatal jaundice refers to jaundice only in the first 14 days of life and not beyond.

3. Neonatal jaundice may be the result of blood group incompatibility, certain causes of haemolysis, infections, liver disease, bruising and metabolic disorders.

4. Glucose-6-phosphate dehydrogenase deficiency is one common cause of severe neonatal jaundice in Singapore.

5. Unconjugated bilirubin is metabolised in the liver to produce conjugated ('direct') bilirubin, which then passes into the gut and is largely excreted in stool.

6. Raised bilirubin levels in the body, or hyperbilirubinaemia, is common in the first week of life, affecting $10 \%$ of term babies and $50 \%$ of preterm babies.

7. Breast milk jaundice occurs after the first two weeks of life and may be due to metabolites contained in breast milk that reduce hepatic uptake of unconjugated bilirubin and increase enterohepatic reuptake.

8. Breast milk jaundice is the same as breastfeeding jaundice.

9. Most cases of neonatal jaundice are 'pathological', but often no underlying disease is found.

10. Hyperbilirubinaemia at very high levels can cross the blood-brain barrier and cause both short- and long-term neurological dysfunction (kernicterus or bilirubin encephalopathy).

11. Adequate formula feeding is approximately $150 \mathrm{kcal} / \mathrm{kg} /$ day or about $1-2$ oz every $2-3$ hours for an infant with average birth weight.

12. Routine supplementation with water is beneficial to prevent hyperbilirubinaemia for exclusively breastfed babies and to treat jaundice.

13. A clinical inspection for jaundice by a healthcare professional should be performed in bright and, preferably, natural light within the first 48 hours of life.

14. Neonatal jaundice occurring within 24 hours of life or a rise in total serum bilirubin level of more than $85 \mu \mathrm{mol} / \mathrm{L}(5 \mathrm{mg} / \mathrm{dL})$ per day should be referred to a specialist for further management.

15. Only total serum bilirubin needs to be monitored in a term baby with jaundice beyond 14 days of life who is otherwise clinically well.

16. The definition of conjugated hyperbilirubinaemia may be based upon the absolute value of direct bilirubin (more than $35 \mu \mathrm{mol} / \mathrm{L}$ ) or its relative value (more than $15 \%$ of total serum bilirubin).

17. Any review of babies with neonatal jaundice beyond 14 days of life must include an assessment of the baby's stool colour.

18. Using stool colour charts is unnecessary, as parents in Singapore can consistently differentiate pale stools from normal stools.

19. Opportunistic screening for prolonged jaundice at one month of life should be carried out by healthcare professionals.

20. Any baby with poor nutrition or a nonreassuring history should be referred early for assessment.

True

False

\section{Doctor's particulars:}

Name in full

MCR number

Email address

Specialty:

\section{SUBMISSION INSTRUCTIONS:}

(1) Log on at the SMJ website: http://www.sma.org.sg/publications/smjcurrentissue.aspx and select the appropriate set of questions. (2) Provide your name, email address and MCR number. (3) Select your answers and click "Submit".

RESULTS:

(1) Answers will be published in the SMJ January 2016 issue. (2) The MCR numbers of successful candidates will be posted online at the SMJ website by 4 January 2016. (3) Passing mark is $60 \%$. No mark will be deducted for incorrect answers. (4) The SMJ editorial office will submit the list of successful candidates to the Singapore Medical Council. (5) One CME point is awarded for successful candidates.

Deadline for submission: (November 2015 SMJ 3B CME programme): 12 noon, 28 December 2015. 